\title{
Adaptive Antenna Array Assisted Dynamic Channel Allocation Techniques
}

\author{
Jonathan S. Blogh, Peter J. Cherriman, and Lajos Hanzo, Senior Member, IEEE
}

\begin{abstract}
The performance of base station adaptive antenna arrays (AAAs) is investigated in conjunction with fixed channel allocation (FCA) and dynamic channel allocation (DCA) schemes. Locally distributed DCA arrangements are studied and benchmarked against standard FCA, in the context of both line-of-sight (LOS) and multipath propagation environments. One-, two-, four-, and eight-element AAAs are employed using the sample matrix inversion (SMI) beamforming algorithm, in both the up- and the downlink. In most investigated scenarios, the locally optimized least interference algorithm (LOLIA) exhibited the best overall compromise in terms of a set of combined metrics, such as the forced termination probability, new call blocking probability, and the probability of a low quality access.
\end{abstract}

Index Terms-Adaptive antennas, adaptive array, beamforming, dynamic channel allocation (DCA), network capacity, wireless networks.

\section{BACKGROUND}

C ELLULAR networks are typically interference limited, where the cochannel interference (CCI) arises from cellular frequency reuse, limiting the quality and capacity of wireless networks [1], [2]. However, adaptive antenna arrays (AAAs) are capable of mitigating this CCI, and thus increase the network capacity [3]-[5]. A further approach to improving the network's performance is the employment of dynamic channel allocation (DCA) techniques, which offer substantially improved call-blocking, packet dropping, and grade-of-service performance in comparison to fixed channel allocation (FCA). A range of so-called distributed DCA algorithms were investigated by Chuang et al. [6]. As compromise schemes, locally optimized distributed DCA algorithms were proposed, for example, by Delli Priscoli et al. [7], [8]. Section IV provides a brief performance summary of the various channel allocation schemes based on our previous work [9]. These results suggested for the scenarios considered [9] that the locally optimized least interference algorithm (LOLIA) provided the best overall compromise in network performance terms. The LOLIA always assigns the least interfered channel, unless it is used in the nearest $n$ neighboring cells by another subscriber. Therefore, the larger $n$ is, the more calls are blocked, since there will be less available channels which are not used by the nearest $n$ base stations. The "reuse exclusion zone" parameter $n$

Manuscript received March 29, 1999; revised February 21, 2000 and September 12, 2000. This work was supported in part by the European Community, Brussels, Belgium; Engineering and Physical Sciences Research Council, Swindon, U.K.

The authors are with the Department of Electronics and Computer Science, University of Southampton, SO17 1BJ, U.K. (e-mail: pjc@ecs.soton.ac.uk; 1h@ecs.soton.ac.uk).

Publisher Item Identifier S 0733-8716(01)00827-7. of the algorithm effectively imposes a minimum reuse distance constraint upon the system.

This paper is concerned with the comparative study of FCA and DCA algorithms [6], [10], [11], many of which were proposed and studied in terms of the achievable grade-of-service (GOS) by Chuang et al.in the past few years [6].

In this paper, the performance gains that may be obtained when using base station AAAs are determined, along with further performance measures such as the carried traffic, call blocking, call dropping, the probability of low quality access, and outage. The comparisons are made for a range of channel allocation techniques under identical conditions, where all users seamlessly roam across the simulation area, rather than simply assigning them to arbitrary, uncorrelated, but essentially stationary, random positions, which was often the strategy in the previous literature.

The paper is structured as follows. Section II briefly describes the motivation behind the employment of AAAs in the mobile environment and the associated challenges to be overcome. Section III introduces the experimental conditions, such as performance metrics, the multipath model, and system parameters employed. The performance comparisons of Section IV constitute the bulk of the paper, contrasting the various channel allocation algorithms, both with and without beamforming, in LOS and multipath environments, leading to the conclusions of Section $\mathrm{V}$.

\section{BEAMFORMING}

The reduction of the frequency reuse distance motivated by achieving increased area spectral efficiency typically results in increased levels of CCI. Adaptive antennas can be used to mitigate these problems [3], [4], [12], [13], by exploiting their angular selectivity in terms of the physical separation between cochannel users, in order to increase the network capacity. Since an AAA is capable of receiving signals from one direction, while nulling signals arriving from other directions, it is inherently suited to a CCI-limited cellular network. Thus, a beam may be formed to communicate with the desired mobile, while nulling interfering mobiles [4]. Assuming that each mobile station is uniquely identifiable, it is a relatively simple task to calculate the antenna array's receiver weights, so as to maximize the received signal-to-interference plus noise ratio (SINR). However, due to the generally uncorrelated up- and downlink channels of frequency division duplexing (FDD), the antenna array weights used for the uplink are not suitable for the downlink. Therefore, it has been proposed to use a feedback loop from the mobile station to the base station, thus allowing the transmit weights of the base station antenna to be 
TABLE I

NETWORK SiMULATION PARAMETERS

\begin{tabular}{l|r||l|r}
\hline Parameter & Value & Parameter & Value \\
\hline \hline Noisefloor & $-104 \mathrm{dBm}$ & Multiple Access & FDMA/TDMA \\
Frame duration & $0.4615 \mathrm{~ms}$ & Cell radius & $218 \mathrm{~m}$ \\
BS transmit power & $10 \mathrm{dBm}$ & MS transmit power & $10 \mathrm{dBm}$ \\
BS power control & No & MS power control & No \\
Number of basestations & 49 & Handover hysteresis & $2 \mathrm{~dB}$ \\
Outage SINR threshold & $17 \mathrm{~dB}$ & Re-allocation SINR threshold & $21 \mathrm{~dB}$ \\
Modulation scheme & $4 \mathrm{QAM}$ & Pathloss exponent & -3.5 \\
Number of timeslots & 8 & Number of carriers & 7 \\
Average inter-call-time & $300 \mathrm{~s}$ & Max new-call queue-time & $5 \mathrm{~s}$ \\
Average call duration & $60 \mathrm{~s}$ & Reference signal modulation & BPSK \\
Beamforming algorithm & SMI & Reference signal length & $8 \mathrm{bits}$ \\
MS speed & $30 \mathrm{mph}$ & Number of antenna elements & $2,4 \& 8$ \\
Pathloss at 1m reference point & $0 \mathrm{~dB}$ & Shadow fading & No \\
\hline
\end{tabular}

adjusted [14], [15] in order to best serve the mobile station. Alternatively, using time division duplexing (TDD) with the corresponding up- and downlink slots being adjacent in time allows the complex conjugate of the receive antenna weights to be used as the transmit weights [4].

\section{SIMULATION CONDITIONS}

\section{A. Performance Metrics}

There are several metrics that can be used to quantify the performance or quality of service provided by a particular channel allocation algorithm. The following performance metrics have been widely used in the literature and were also advocated by Chuang et al. [6].

- New call blocking probability $P_{B}$ as a result of insufficient frequency-timeslot combinations or as a consequence of low up- or down-link SINR levels.

- Call dropping or forced termination probability, $P_{D}$ or $P_{F T}$, due to poor up- or downlink SINR levels.

- Probability of low quality connection $P_{\text {low }}$ quantifying the chances that either the up- or downlink signal quality is below the level required by the specific transceiver to maintain our target performance of 5\% frame error rate (FER) [16].

- Probability of outage $P_{\text {out }}$ is defined as the probability that the lower of the up- and downlink SINR values is below the threshold at which the call is deemed to be in outage, i.e., $10 \%$ FER. A prolonged outage results in the call being dropped.

A handover or handoff event occurs when the quality of the communications channel used degrades, and hence the call is switched to a newly allocated channel. If the new channel belongs to the same base station, this is referred to as an intracell handover. Generally, an intracell handover is performed, either when the channel quality degrades due to CCI or in order to increase the system's performance and capacity. Intercell handovers occur typically when the mobile approaches the fringes of the cell, and hence, the signal strength degrades, requiring a handover to a nearer base station.

\section{B. System Parameters}

The performance of the various channel allocation algorithms was investigated in a microcellular system, the parameters of which are defined in Table I. The system used eight time slots but with only seven carrier frequencies assigned to the whole network, in order to maintain an acceptable computational load. This implied that the DCA system could theoretically handle a maximum of $7 \times 8=56$ simultaneous calls at one base station. If a channel allocation request for a new call could not be satisfied immediately, it was queued for up to $5 \mathrm{~s}$. After this queuing period, the call was classed as blocked unless it was admitted to the network. The network was synchronous from cell to cell, thus channels on different time slots of the same frequency were orthogonal. The call arrivals were Poisson-distributed, and hence, the call duration and intercall periods were exponentially distributed [11], [17], with the mean values shown in Table I.

The activity rate of the users was fairly high, enabling us to expedite our experiments. The mobiles were capable of moving freely, in random directions, at a speed of $30 \mathrm{mph}$ within the simulation area, which comprised 49 cells, with a cell radius of $218 \mathrm{~m}$. In our initial work [9], we experienced some border or edge effects near the fringes of the 49-cell simulation area, which resulted in the central cells experiencing more unfavorable conditions than the edge cells since there were no mobiles roaming outside of the 49-cell boundary. Hence, near the 49-cell boundary, a reduced interference load was experienced. In order to avoid this problem, a tessellating rhombic simulation area of seven cells by seven cells was used, thus allowing the simulation area to be replicated around itself. More explicitly, mobile stations and their signals were "wrapped around" from one side of the network to the other [18], [19]. Hence, for example, a mobile station in call which left the network at its edge reentered the network on the opposite side, while inflicting CCI to all users which may be located at either edge of the network. 
The handover process was modeled using the "reallocation SINR," defined as the average SINR required by a QPSK/4QAM transceiver in order to maintain a 5\% transmission FER over a narrowband Rayleigh fading channel. When the signal quality expressed in terms of the SINR drops below this level, a low quality access is encountered and the mobile requests a new physical channel to handover to. If, while waiting for a reallocation handover, which typically involves waiting for a free slot to become available, the signal quality drops further, namely below the "outage SINR," defined as the SINR required to maintain a $10 \%$ FER, then a signal outage occurs. A prolonged outage leads to the call being dropped. Since a dropped call is less desirable from the user's viewpoint than a blocked call, a handover queueing system (HQS) was employed. By forming a queue of the handover requests, which have a higher priority during contention for network resources than new calls, it is possible to reduce the number of dropped calls at the expense of the blocked call probability. A further advantage of the HQS is that a time window is formed during which the handover may take place. This handover time window enables the user to wait for a slot to become free. This increases the chance of a successful handover.

The receiver antenna array weights were calculated using the sample matrix inversion (SMI) algorithm [5], [20], [21], using a binary phase shift keying (BPSK) modulated reference signal of eight bits. The reference signal length was chosen as a compromise between complexity, the number of interferers that may be assigned uncorrelated reference signals, and the estimation quality of the covariance matrix of the array's output signal. One of the eight reference signals was assigned to the desired mobile and the remaining seven were allocated to the interfering mobiles.

Line-of-sight (LOS) and multipath environments without shadow fading were considered. The up- and downlink channels were assumed to be identical, thus allowing the same antenna pattern to be used in both the up- and the downlinks, as in a TDD system exhibiting similar up- and downlink interference. This assumption of channel predictability should, therefore, give an upper limit to the performance gains that may be achieved using an adaptive array. Having described the beamforming parameters, in the next section, we present the parameters of the multipath environment used.

In order to render the simulations realistic, we used two multipath rays, in addition to the LOS ray, each having one-third of the direct-path's power. The angle-of-arrival of each multipath ray was determined using the so-called geometrically based single-bounce elliptical model (GBSBEM) of [22], [23] with parameters chosen such that the multipath rays had one-third of the received power of the direct ray. The probability density function (PDF) of the angle-of-arrival distribution used in the simulations generated using the GBSBEM is shown in Fig. 1. It was assumed that the multipath rays arrived with no time delay relative to the LOS path. However, in a practical system, a space-time equalizer [24], [25] would be required to prevent the nulling of the delayed paths.

Having described the simulation parameters, in the next section we present our simulation results, quantifying the amount of traffic that can be supported by each channel allocation algorithm.

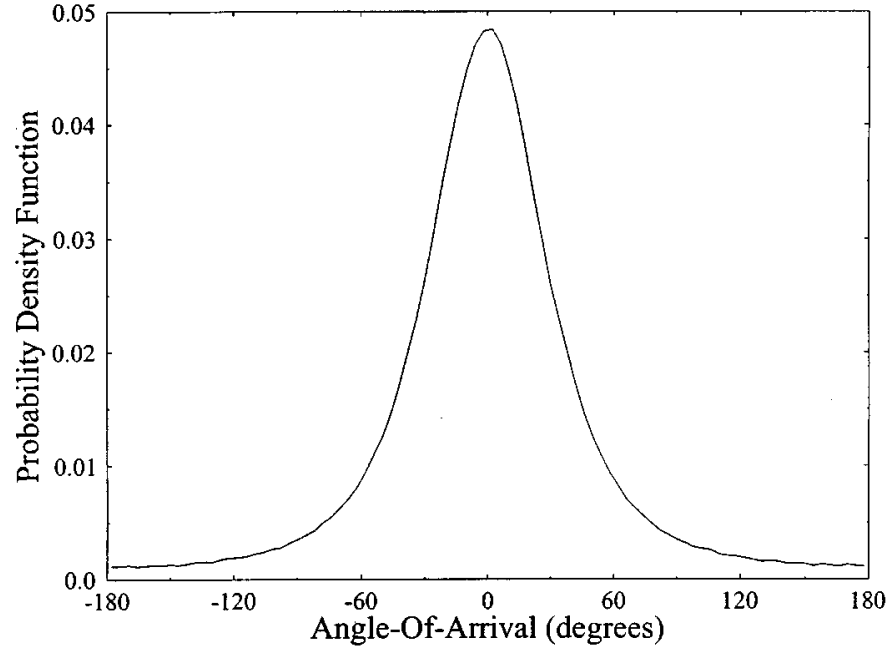

Fig. 1. Probability density function of angle-of-arrival of the multipath rays, centered about the angle-of-arrival of the line-of-sight path.

TABLE II

MAXimum Number of MobiLE USERS THAT CAN BE SUPPORTED by THE VARIOUS DCA ALGORITHMS

\begin{tabular}{|c|c|c|}
\hline \multirow[b]{2}{*}{ Algorithm } & \multicolumn{2}{|c|}{ Number of users supported by network } \\
\hline & $\begin{array}{c}\text { Conservative } \\
P_{F T}=1 \%, P_{\text {low }}=1 \%, P_{B}=3 \%\end{array}$ & $\begin{array}{c}\text { Lenient } \\
P_{F T}=1 \%, P_{\text {low }}=2 \%, P_{B}=5 \%\end{array}$ \\
\hline FCA & 820 & 1120 \\
\hline HTA & 1435 & 1520 \\
\hline LFA & 1555 & 1705 \\
\hline LOMIA $(n=19)$ & 1505 & 2040 \\
\hline LTA & 1815 & 1830 \\
\hline LIA & 1820 & 1820 \\
\hline LOLIA $(n=7)$ & 1860 & 2115 \\
\hline LOLIA $(n=19)$ & 1935 & 2005 \\
\hline
\end{tabular}

\section{Performance Study}

The results presented here are the combination of adaptive beamforming at the base station and fixed as well as DCA algorithms.

The results in Table II indicate the achievable network capacities, without AAAs and shadow fading, for various DCA algorithms and the FCA algorithm. Observe at the top of Table II that we used both a set of conservative and lenient network quality requirements expressed in terms of $P_{F T}, P_{\text {low }}$, and $P_{B}$ as defined explicitly in Section IV-C. These results are based on a summary of our previous work [9]. Below we briefly define the various DCA algorithms. The least interference algorithm (LIA) [6] always assigns the channel suffering from the least interference, which minimizes the system's interference load. A more advanced version of this scheme is the least interference below threshold algorithm (LTA) [6], which attempts to maintain the amount of interference below a given threshold. The threshold is determined by the transceiver's interference rejection tolerance expressed in terms of the SINR required for attaining its target FER. In contrast, the highest interference below threshold algorithm (HTA) [6] allocates the most interfered channel, whose interference is below the maximum acceptable level. A technique 


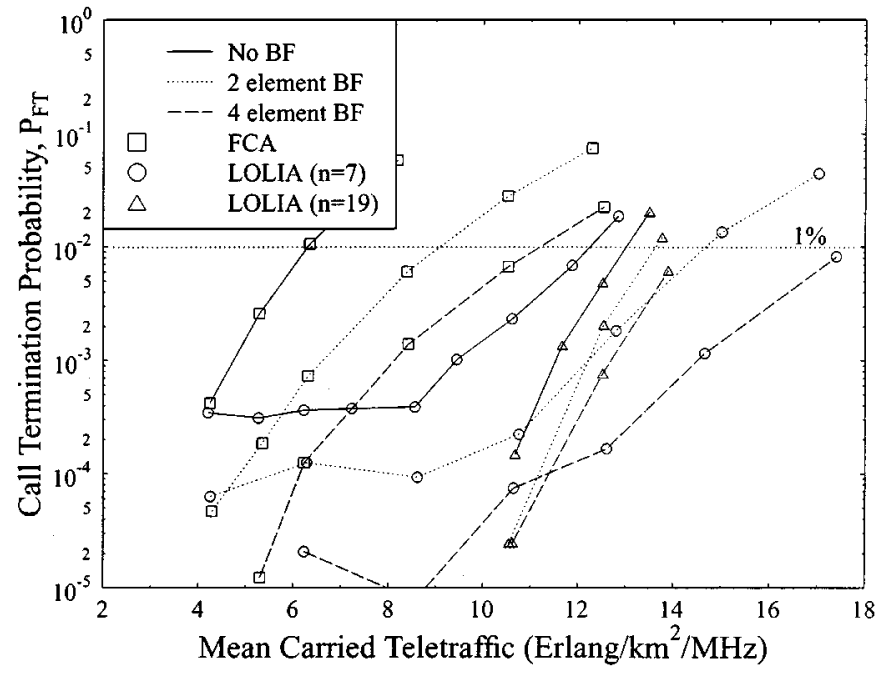

Fig. 2. Dropping probability performance versus mean carried traffic, for the LOLIA, with 7 and 19 "local" base stations, and FCA employing a 7-cell reuse cluster, under uniform traffic, for a single antenna element, as well as for 2 and 4 element antenna arrays with beamforming in an LOS environment. The corresponding multipath performance curves are portrayed in Fig. 4.

similar to the LTA is the lowest frequency below threshold algorithm (LFA) [6], which attempts to reduce the number of carrier frequencies in use simultaneously, while allocating the least interfered channel exhibiting an interference level below the given threshold. Finally, the locally optimized most interference algorithm (LOMIA) selects the most interfered channel, rather than the least interfered one, as in the LOLIA.

\section{A. Comparing the LOLIA to FCA for LOS Scenario}

First, we compared the FCA and the LOLIA DCA under uniform traffic distribution conditions using both a single antenna element and AAAs consisting of two and four elements in an LOS propagation environment. The FCA scheme employed a seven-cell reuse cluster, corresponding to one carrier frequency per base station. The LOLIA was used with the constraints of 7 and 19 nearest base stations, i.e., $n=7$ or 19 .

Fig. 2 shows that, as expected, the FCA algorithm performed the least satisfactorily of the three channel allocation schemes investigated, with respect to its call dropping performance. Even in conjunction with a four-element AAA, it exhibited a higher call dropping rate than that of either of the LOLIAs ( $n=19$ and $n=7$ ). The large exclusion zone of the LOLIA using $n=19$ led to a low dropping probability of less than $1 \times 10^{-3}$ for teletraffic loads below approximately 12 Erlang $/ \mathrm{km}^{2} / \mathrm{MHz}$. However, the rapid rise in the call dropping probability upon increasing the teletraffic became unacceptable for teletraffic loads in excess of about 13 Erlang $/ \mathrm{km}^{2} / \mathrm{MHz}$. The large exclusion zone of the algorithm prevented handovers occurring, since there were no free channels available in the vicinity, hence resulting in a high number of dropped calls. Thus, for $n=19$, the employment of AAAs at the base stations did not improve the performance significantly, unlike for the FCA and LOLIA using $n=7$, which were predominantly interference limited. The call dropping performance of the LOLIA using $n=7$ benefited the most from the assistance of

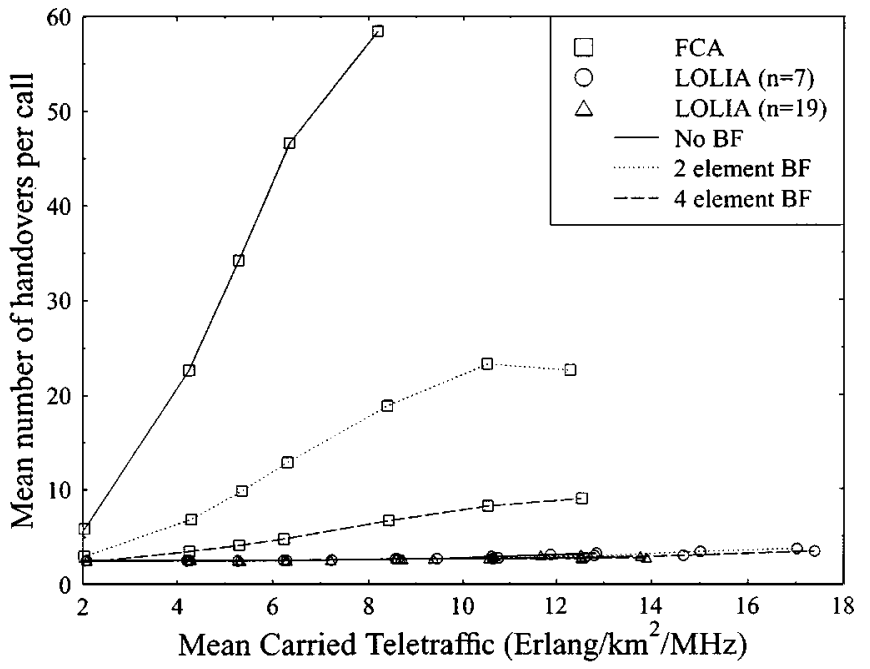

Fig. 3. Mean number of handovers per call versus mean carried traffic, for comparison of the LOLIA, with 7 and 19 "local" base stations, and of FCA employing a 7-cell reuse cluster, under uniform traffic, for a single antenna element, as well as for 2 and 4 element antenna arrays with beamforming in an LOS environment. The corresponding multipath performance curves are portrayed in Fig. 5.

AAAs, with the greatest gains in call dropping performance at the higher teletraffic levels.

The LOLIA using $n=19$ offered the worst call blocking performance (not explicitly shown) of the three channel allocation schemes, with the AAAs having little effect. This demonstrated that the limiting factor was not inadequate signal quality for a call to be set up but the lack of available frequency-time slot combinations due to the large exclusion zone. The FCA algorithm benefited only to a limited extent from the employment of the AAAs, suggesting that the majority of the blocked calls were as a result of the limited availability of frequency/time slot combinations. Inadequate signal quality caused the remainder of the blocked calls. The call blocking performance of the LOLIA using $n=7$ appeared mainly to be interference limited, hence the AAAs resulted in a significant reduction of the number of blocked calls, particularly for mean carried traffic levels in excess of 9 Erlang $/ \mathrm{km}^{2} / \mathrm{MHz}$.

The effect of beamforming on the number of handovers performed can be seen in Fig. 3. The LOLIAs required the least frequent handovers, with beamforming barely altering the results. By contrast, the number of handovers performed when using the FCA algorithm was reduced significantly due to employing AAAs, with a maximum reduction of $72 \%$ for two elements, and $90 \%$ for four elements. This translates into a significantly reduced signaling load for the network, since it has to carry out far less handovers, therefore reducing the complexity of the network infrastructure.

\section{B. Comparing the LOLIA to FCA Over Multipath Channels}

Following our previous experiments, where a purely LOS environment existed between the mobiles and their base stations, this section presents results for a multipath environment using two-, four-, and eight-element AAAs.

Comparing the call blocking probabilities of the multipath environment to those of the LOS environment (neither explicitly 


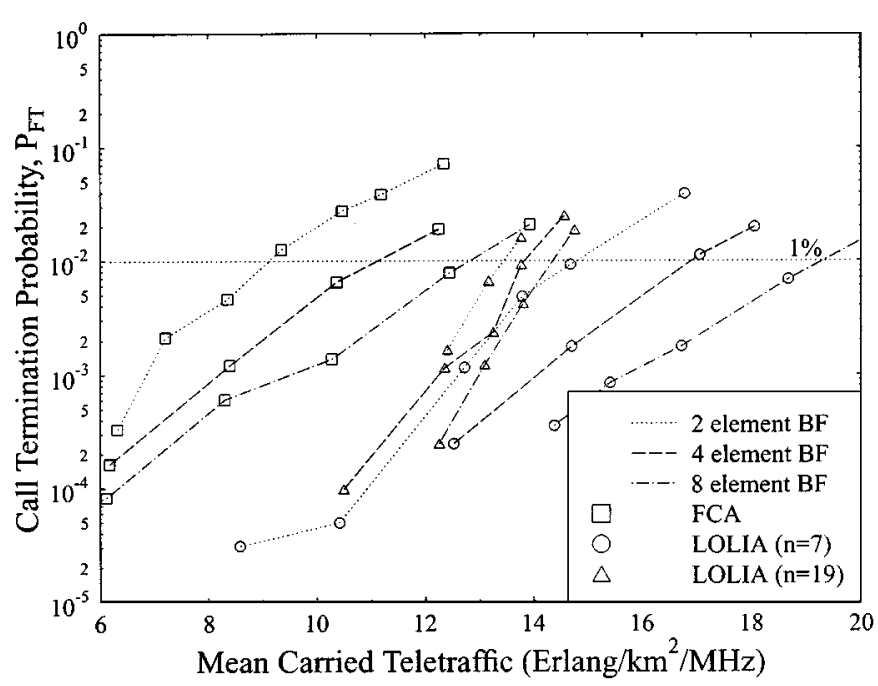

Fig. 4. Call dropping probability performance versus mean carried traffic, for comparison of the LOLIA, with 7 and 19 "local" base stations, and of FCA using a 7-cell reuse cluster, under uniform traffic, for 2, 4, and 8 element antenna arrays with beamforming in a multipath environment. The corresponding LOS performance curves are portrayed in Fig. 2.

shown) revealed that all of the channel allocation algorithms behave similarly for both radio environments. The FCA scheme actually behaved more unfavorably in terms of its new call blocking probability, as the number of AAA elements was increased. However, this is a result of the additional antenna elements improving the other performance measures, such as the call dropping rate. This enabled additional calls to be sustained at a given time, leading to a higher call blocking rate. In conjunction with an exclusion zone of 19 cells, we found that the LOLIA's blocking performance was barely affected by the AAAs, while for $n=7$ the blocked call rate was improved by a factor of 10 (at $14-17$ Erlang $/ \mathrm{km}^{2} / \mathrm{MHz}$ ) although, again, these results were not shown here diagrammatically due to space constraints.

Fig. 4 shows the probability of a dropped call in a multipath propagation environment, which was slightly higher than for the LOS scenario of Fig. 2, when considered in the context of a given channel allocation algorithm and for a given antenna array size. The call dropping rate was improved with the aid of AAAs for all of the channel allocation algorithms, although the LOLIA using $n=19$ did not benefit to the same extent as the other algorithms.

Fig. 5 demonstrates the significant impact of AAAs on the mean number of handovers per call for the FCA algorithm in a multipath environment. Even in conjunction with AAAs, more handovers per call were invoked, when using the FCA system, than for either of the LOLIAs using a single antenna element. Furthermore, for a given size of AAA, a higher number of handovers was required in the multipath environment than in the LOS scenario. The LOLIAs ( $n=7$ and $n=19$ ) required significantly fewer handovers than the FCA, regardless of the propagation environment, and did not benefit from the AAAs in terms of the required handovers per call.

\section{Overview of Results and Discussion}

In this paper, we have investigated the teletraffic performance of the FCA and LOLIA with the assistance of base station AAAs

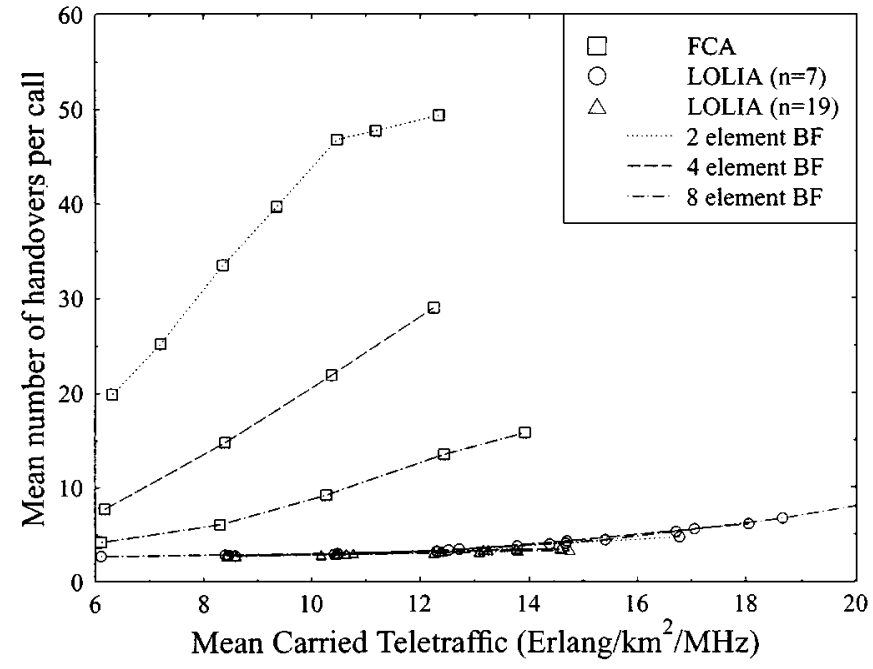

Fig. 5. Mean number of handovers per call versus mean carried traffic, for comparison of the LOLIA, with 7 and 19 "local" base stations, and of FCA using a 7-cell reuse cluster, under uniform traffic, for 2,4 , and 8 element antenna arrays with beamforming in a multipath environment. The corresponding LOS performance curves are portrayed in Fig. 3.

for both LOS and multipath propagation environments without shadow fading. None of the algorithms performed best in terms of every performance metric. Therefore, in order to compare our results for the various channel allocation schemes, it is necessary to consider a combination of performance metrics since an algorithm may provide excellent performance in terms of one metric but poor performance in terms of another. Therefore, we defined a conservative and a lenient scenario, as follows [9].

- Conservative Scenario: $P_{B} \leq 3 \%, P_{\text {low }} \leq 1 \%$ and $P_{F T} \leq 1 \%$.

- Lenient Scenario: $P_{B} \leq 5 \%, P_{\text {low }} \leq 2 \%$ and $P_{F T} \leq 1 \%$. It can be seen from Table III that in an LOS environment all of the channel allocation schemes benefit from the use of base station AAAs in terms of an increased level of teletraffic carried, hence supporting an increased number of users. The FCA algorithm benefited most from the employment of AAAs with a $160 \%$ increase in terms of the number of users supported, when using a four-element antenna array. The performance improvements of the LOLIA with $n=7$ due to using AAAs were more modest than for the FCA system. Specifically, 44\% more users were supported by the four-element AAA assisted LOLIA using $n=7$, when compared to the single antenna element based results. The network capacity of the LOLIA with a 19-cell exclusion zone was higher than that of the LOLIA using $n=7$, until the limited number of channels available in conjunction with such a large exclusion zone became significant. Up to this point, the AAAs reduced the levels of interference, thus improving the network capacity. However, when using a four-element AAA, the new call blocking probability became the dominant network performance factor.

Table IV presents similar results to Table III but for a multipath environment, with the bold values highlighting the AAA sizes common to both sets of investigations.

From this table, it can be seen that the LOLIA using $n=19$ carries approximately the same traffic in the multipath scenario, which translates into a similar network capacity to that of the 
TABLE III

MaXimum Mean Carried Traffic, and MaXimum Number of Mobile Users that Can be Supported by Each Configuration, whiLE MeEting the

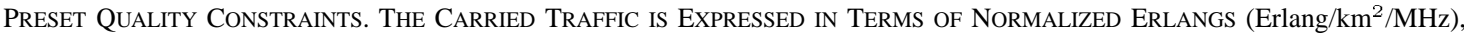
FOR THE NETWORK DESCRIBED IN TABLE I IN AN LOS ENVIRONMENT

\begin{tabular}{|c|c|c|c|c|c|c|}
\hline \multirow[b]{2}{*}{ Algorithm } & \multicolumn{3}{|c|}{$\begin{array}{c}\text { Conservative } \\
P_{F T}=1 \%, P_{\text {low }}=1 \%, P_{B}=3 \%\end{array}$} & \multicolumn{3}{|c|}{$\begin{array}{c}\text { Lenient } \\
P_{F T}=1 \%, P_{\text {low }}=2 \%, P_{B}=5 \%\end{array}$} \\
\hline & Users & Traffic & $\begin{array}{l}\text { Limiting } \\
\text { Factor }\end{array}$ & Users & Traffic & $\begin{array}{l}\text { Limiting } \\
\text { Factor }\end{array}$ \\
\hline FCA, 1 element & 340 & 3.6 & $P_{l o w}$ & 465 & 4.9 & $P_{\text {low }}$ \\
\hline FCA, 2 elements & 575 & 6.1 & $P_{l o w}$ & 755 & 7.9 & $P_{\text {low }}$ \\
\hline FCA, 4 elements & 885 & 9.3 & $P_{\text {low }}$ & 1105 & 11.2 & $P_{F T}$ \\
\hline LOLIA $(\mathrm{n}=7), 1$ element & 990 & 10.5 & $P_{\text {low }}$ & 1065 & 11.45 & $P_{\text {low }}$ \\
\hline LOLIA $(\mathrm{n}=7), 2$ elements & 1155 & 12.35 & $P_{\text {low }}$ & 1260 & 13.5 & $P_{\text {low }}$ \\
\hline LOLIA $(n=7), 4$ elements & 1420 & 14.9 & $P_{\text {low }}$ & 1535 & 16.5 & $P_{\text {low }}$ \\
\hline LOLIA $(n=19), 1$ element & 1020 & 10.9 & $P_{\text {low }}$ & 1090 & 11.6 & $P_{\text {low }}$ \\
\hline LOLIA $(n=19), 2$ elements & 1200 & 12.5 & $P_{\text {low }}$ & 1330 & 13.35 & $P_{l o w}$ \\
\hline LOLIA $(n=19), 4$ elements & 1335 & 13.45 & $P_{B}$ & 1400 & 13.9 & $P_{B}$ \\
\hline
\end{tabular}

TABLE IV

Maximum Mean Carried Traffic, and MaXimum Number of Mobile Users that Can be Supported by Each Configuration, while MeEting the

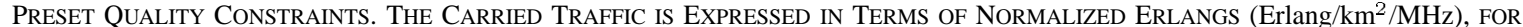
THE NeTWork Described in TABLE I in a Multipath ENVIRONMENT

\begin{tabular}{|c|c|c|c|c|c|c|}
\hline \multirow[b]{2}{*}{ Algorithm } & \multicolumn{3}{|c|}{$\begin{array}{c}\text { Conservative } \\
P_{F T}=1 \%, P_{\text {low }}=1 \%, P_{B}=3 \%\end{array}$} & \multicolumn{3}{|c|}{$\begin{array}{c}\text { Lenient } \\
P_{F T}=1 \%, P_{l o w}=2 \%, P_{B}=5 \%\end{array}$} \\
\hline & Users & Traffic & $\begin{array}{l}\text { Limiting } \\
\text { Factor }\end{array}$ & Users & Traffic & $\begin{array}{l}\text { Limiting } \\
\text { Factor }\end{array}$ \\
\hline FCA, 2 element & 600 & 6.0 & $P_{\text {low }}$ & 740 & 7.65 & $P_{\text {low }}$ \\
\hline FCA, 4 elements & 790 & 8.3 & $P_{\text {low }}$ & 995 & 10.3 & $P_{\text {low }}$ \\
\hline FCA, 8 elements & 1085 & 11.2 & $P_{\text {low }}$ & 1250 & 12.8 & $P_{F T}$ \\
\hline LOLIA $(n=7), 2$ element & 1195 & 12.65 & $P_{\text {low }}$ & 1290 & 13.7 & $P_{l o w}$ \\
\hline LOLIA $(n=7), 4$ elements & 1370 & 14.35 & $P_{\text {low }}$ & 1475 & 15.6 & $P_{\text {low }}$ \\
\hline LOLIA $(\mathrm{n}=7), 8$ elements & 1555 & 16.15 & $P_{\text {low }}$ & 1700 & 17.7 & $P_{\text {low }}$ \\
\hline LOLIA $(n=19), 2$ element & 1235 & 12.65 & $P_{\text {low }}$ & 1325 & 13.3 & $P_{\text {low }}$ \\
\hline LOLIA $(n=19), 4$ elements & 1360 & 13.55 & $P_{B}$ & 1410 & 13.8 & $P_{F T}$ \\
\hline LOLIA $(n=19), 8$ elements & 1385 & 13.7 & $P_{B}$ & 1475 & 14.15 & $P_{B}$ \\
\hline
\end{tabular}

LOS scenario of Table III. Again, the number of users supported by the network is limited by the probability of a low quality access and the new call blocking probability. The performance of the LOLIA using $n=7$ is interference limited, with the smaller reuse distance or exclusion zone leading to many sources of relatively strong interference, all requiring interference cancellation. Hence, as the number of AAA elements increased, so did the number of users supported, with an average improvement of about $15 \%$ for each doubling of the number of array elements.

\section{CONCLUSION}

In this paper, we have examined the FCA and LOLIA channel allocation schemes assisted by AAAs, employed at the base station for the up- and the downlink using identical transmit and receive beam patterns, and compared their performance in non- shadow faded LOS and multipath propagation environments, using the performance metrics of Section III-A. Under the current reasonable set of assumptions, the following conclusions were drawn and further research will be targeted at verifying them under practical scenarios. The FCA algorithm was found to benefit most from the employment of AAAs for both the LOS and multipath scenarios. However, the LOLIA using $n=7$ offered a higher network capacity than FCA under identical assumptions, although benefiting less from the use of AAAs. For example, the LOLIA using $n=7$ assisted by a two-element array supported a higher number of users than the FCA-based network using an eight-element AAA. The LOLIA using $n=$ 19 offered a smaller performance improvement, when using AAAs but the large reuse distance limited its impact. Our future work is dedicated to studying the effects of power control and burst-by-burst adaptive modulation techniques [26]. 


\section{ACKNOWLEDGMENT}

The rigorous critique of the anonymous reviewers is gratefully acknowledged.

\section{REFERENCES}

[1] T. Kanai, "Autonomous reuse partitioning in cellular systems," in IEEE Proc. Veh. Technol. Conf., vol. 2, 1992, pp. 782-785.

[2] I. Katzela and M. Naghshineh, "Channel assignment schemes for cellular mobile telecommunication systems: A comprehensive survey," IEEE Pers. Commun. Mag., vol. 3, pp. 10-31, June 1996.

[3] J. Litva and T. Lo, Digital Beamforming in Wireless Communications. London: Artech House, 1996.

[4] L. C. Godara, "Applications of antenna arrays to mobile communications-Part I: Performance improvement, feasibility, and system considerations," Proc. IEEE, vol. 85, pp. 1029-1060, July 1997.

[5] _ "Applications of antenna arrays to mobile communications-Part II: Beam-forming and direction-of-arrival considerations," Proc. IEEE, vol. 85, pp. 1193-1245, Aug. 1997.

[6] M. M. L. Cheng and J. C. I. Chuang, "Performance evaluation of distributed measurement-based dynamic channel assignment in local wireless communications," IEEE J Select. Areas Commun., vol. 14, pp. 698-710, May 1996.

[7] A. Baiocchi, F. D. Priscoli, F. Grilli, and F. Sestini, "The geometric dynamic channel allocation as a practical strategy in mobile networks with bursty user mobility," IEEE Trans. Veh. Technol., vol. 44, pp. 14-23, Feb. 1995.

[8] F. D. Priscoli, N. P. Magnani, V. Palestini, and F. Sestini, "Application of dynamic channel allocation strategies to the GSM cellular network," IEEE J. Select. Areas Commun., vol. 15, pp. 1558-1567, Oct. 1997

[9] P. Cherriman, F. Romiti, and L. Hanzo, "Channel allocation for thirdgeneration mobile radio systems," in Proc. ACTS, Rhodes, Greece, June 1998, pp. 255-260.

[10] J. C. I. Chuang, "Performance issues and algorithms for dynamic channel assignment," IEEE J. Select. Areas Commun., vol. 11, pp. 955-963, Aug. 1993.

[11] J. C. I. Chuang and N. R. Sollenberger, "Performance of autonomous dynamic channel assignment and power control for TDMA/FDMA wireless access," IEEE J. Select. Areas Commun., vol. 12, pp. 1314-1323, Oct. 1994.

[12] R. Kohno, "Spatial and temporal communication theory using adaptive antenna array," IEEE Pers. Commun., vol. 5, pp. 28-35, Feb. 1998.

[13] J. H. Winters, "Smart antennas for wireless systems," IEEE Pers. Commun., vol. 5, pp. 23-27, Feb. 1998.

[14] D. Gerlach and A. Paulraj, "Adaptive transmitting antenna arrays with feedback," IEEE Signal Process. Lett., vol. 1, pp. 150-152, Oct. 1994.

[15] _ _ "Base station transmitting antenna arrays for multipath environments," Signal Processing, pp. 59-73, 1996.

[16] P. Cherriman and L. Hanzo, "Programmable H.263-based wireless video transceivers for interference-limited environments," IEEE Trans. Circuits Syst. Video Technol., vol. 8, pp. 275-286, June 1998.

[17] A. S. Tanenbaum, "Introduction to queueing theory," in Computer Networks, 2nd ed. Englewood Cliffs, NJ: Prentice-Hall, 1989, pp. 631-641.

[18] G. J. Foschini and Z. Miljanic, "Distributed autonomous wireless channel assignment algorithm with power control," IEEE Trans. Veh. Technol., vol. 44, pp. 420-429, Aug. 1995.

[19] J. C.-I. Chuang and N. R. Sollenberger, "Spectrum resource allocation for wireless packet access with application to advanced cellular internet service," IEEE J. Select. Areas. Commun., vol. 16, pp. 820-829, Aug. 1998

[20] I. Reed, J. Mallett, and L. Brennan, "Rapid convergence rate in adaptive arrays," IEEE Trans. Aerosp. Electron. Syst., vol. AES-10, pp. 853-863, Nov. 1974.

[21] R. Monzingo and T. Miller, Introduction to Adaptive Arrays. New York: Wiley, 1980.

[22] J. Liberti and T. Rappaport, "A geometrically based model for line-ofsight multipath radio channels," in Proc. Veh. Technol. Conf., 1996, pp. 844-848.
[23] R. Ertel, P. Cardieri, K. Sowerby, T. Rappaport, and J. Reed, "Overview of spatial channel models for antenna array communications systems," IEEE Pers. Commun., pp. 10-22, Feb. 1998.

[24] R. Kohno, "Spatial and temporal communication theory using software antennas for wireless communications," in Wireless Communications TDMA versus CDMA. Norwell, MA: Kluwer, 1997, pp. 293-321.

[25] Y. Ogawa and T. Ohgane, "Adaptive antennas for future mobile radio," IEICE Trans. Fundam., vol. E79-A, pp. 961-967, July 1996.

[26] L. Hanzo, W. T. Webb, and T. Keller, Single- and Multi-Carrier Quadrature Amplitude Modulation. New York: Wiley, IEEE, 2000

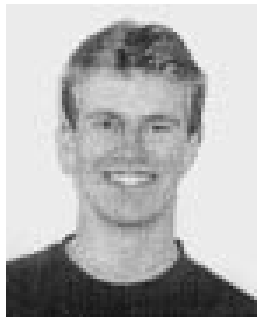

Jonathan S. Blogh received the M.Eng. degree in 1997 (with distinction) in information engineering from the University of Southampton, Southampton, U.K. Since 1997, he has been pursuing the Ph.D. degree in mobile communications at the University of Southampton.

His current areas of research include the networking aspects of FDD and TDD mode third-generation mobile cellular networks.

Mr. Blogh was awarded the IEE Lord Lloyd of Kilgerran Memorial Prize for interest and commitment to mobile radio and RF engineering in 1997.

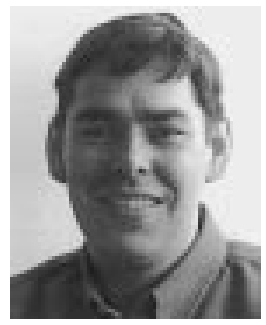

Peter J. Cherriman received the M.Eng. degree in information engineering from the University of Southampton, Southampton, U.K. in 1994. Since 1994, he has been pursuing the Ph.D. degree in mobile video networking with the Department of Electronics and Computer Science at the University of Southampton.

Currently he is working on projects for the Mobile Virtual Centre of Excellence. His current areas of research include robust video coding, microcellular radio systems, power control, dynamic channel allocation, and multiple access protocols.

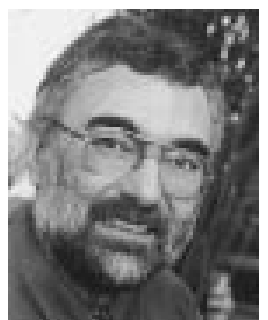

Lajos Hanzo (SM'92) received the Dipl. Ing. degree in electronics in 1976 and the Ph.D. degree in 1983 , both from the Technical University of Budapest, Hungary

During his 24-year career in telecommunications, he has held various research and academic posts in Hungary, Germany, and the U.K. Since 1986, he has been with the Department of Electronics and Computer Science, University of Southampton, Southampton, U.K., and has been a Consultant to Multiple Access Communications Ltd., Southampton, U.K. Currently he holds a chair in telecommunications. He co-authored five books on mobile radio communications, published about 300 research papers, organized and chaired conference sessions, presented overview lectures, and was awarded a number of distinctions. Currently he is managing an academic research team, working on a range of research projects in the field of wireless multimedia communications sponsored both by industry and under the auspices of the Engineering and Physical Sciences Research Council (EPSRC), U.K., the European IST Programme, and the Mobile Virtual Centre of Excellence (VCE), U.K. For further information on research in progress and associated publications, please refer to http://www-mobile.ecs.soton.ac.uk. 